\title{
THE NON-DYSTROPHIC MYOPATHIES
}

\author{
John Pearce, M.B., M.R.C.P., \\ Department of Neurology, The General Infirmary, \\ Leeds.
}

THE TERM 'myopathy' is applied to any disorder of the muscle fibre, the muscle fibre membrane, the myoneural junction, or the muscle connective tissue. 'Non-dystrophic myopathy' includes all diseases of muscle excluding those genetically determined primary degenerative myopathies, collectively known as Muscular Dystrophy.

TABLE 1

Classification of Non-Dystrophic Myopathies

1. Inflammatory Polymyositis

Myopathy Connective tissue disorders

Other inflammatory myopathies

2. Metabolic

Myopathy

Familial periodic paralysis

Muscle glycogenoses

Myoglobinuric myopathies

Myopathies associated with electrolyte imbalance

Unclassified myopathies

\begin{tabular}{|c|c|}
\hline $\begin{array}{l}\text { 3. Endocrine } \\
\text { Myopathy }\end{array}$ & $\begin{array}{l}\text { Thyrotoxicosis } \\
\text { Cushing's Syndrome } \\
\text { Steroid myopathy } \\
\text { Hypothyroidism } \\
\text { Hypoadrenalism } \\
\end{array}$ \\
\hline $\begin{array}{l}\text { 4. Neoplastic } \\
\text { Myopathy }\end{array}$ & $\begin{array}{l}\text { Carcinoma } \\
\text { Reticuloses }\end{array}$ \\
\hline $\begin{array}{l}\text { 5. The Myasthenic } \\
\text { Syndrome }\end{array}$ & $\begin{array}{l}\text { Myasthenia gravis } \\
\text { Thyrotoxic myasthenia } \\
\text { Myopathic-myasthenic } \\
\text { syndrome of carcinoma }\end{array}$ \\
\hline
\end{tabular}

Inflammatory Myopathies

Polymyositis

This term encompasses a group of inflammatory disorders of muscle of varied aetiology. In some patients the condition is confined to muscle tissues but in most instances there is evidence of some systemic disturbance indicating that the myopathy is only part of a more generalised disease of connective tissues. Accordingly, polymyositis is classified on a clinical basis, until our understanding of this group of disorders as a whole is more complete.

\section{TABLE 2}

Classification of Inflammatory Myopathies (Modified from Walton and Adams, 1958)

(i) Polymyositis with purely muscle involvementAcute, Subacute, Chronic.

(ii) Polymyositis with connective tissue disease or dermatomyositis, but muscle involvement dominant.

(iii) Polymyositis with connective tissue disease or dermatomyositis, but major involvement is of primary disease and muscle weakness is not striking.

(iv) Polymyositis complicating malignant disease.
Polymyositis may affect people of any age, but the age of onset is from 30 to 60 in $60 \%$ of cases. The chief symptom is weakness of proximal muscles of the arms and/or legs in every case. Distal muscles are affected in one third, and the neck muscles in two thirds of patients. Fever, muscular pain and tenderness are seen most often in the more acute forms, and their absence should not lead to neglecting polymyositis as a possible diagnosis.

Acute polymyositis is not common, but may progress rapidly and involve the respiratory muscles, sometimes with a fatal termination within a few weeks or months. Subacute and chronic forms are more frequent, and present with a progressive weakness and moderate wasting of shoulder and pelvic girdle muscles. There is sometimes no systemic disturbance, and pain and tenderness are lacking. All proximal muscles tend to be affected, and to an equal extent. In patients with the disease of long standing, fibrous contractures, and calcinosis of subcutaneous and muscle tissues may develop.

Dysphagia, Raynaud's phenomenon, joint pains, and skin rashes are common accompaniments of muscle weakness, and are important clues to the presence of polymyositis. The acuteness of the onset, and the rate of progression are enormously variable, and spontaneous but usually incomplete remissions are occasionally seen.

The skin lesion of dermatomyositis is seen in $40 \%$ of patients (Pearson, 1964). A violaceous erythema is characteristic, and involves particularly the eyelids, face and extensor aspects of the arms. The heliotrope scaling eruption of the upper eyelids is virtually diagnostic. A diffuse erythema and scaling eruptions are common but non-specific. Tightening of the skin over the face, or the distal phalanges, which appear tapered and shiny, and may show small telangiectatic patches, are characteristic of the skin involvement of systemic sclerosis, of which polymyositis may be symptomatic. In patients of middle or old age, polymyositis or dermatomyositis may be secondary to neoplastic lesions, and this is discussed in a later section. 
Polymyositis can usually be distinguished from muscular dystrophy by the following features: -

(i) Polymyositis is more common in females than males, and spontaneous remissions occur. In childhood dystrophy males predominate, and progression though slower, is usually relentless without a true remission, although periods of temporary arrest occur.

(ii) Muscle involvement is diffuse in polymyositis, but tends to be patchy and selective in dystrophy.

(iii) Weakness of the neck muscles, dysphagia and skin rashes are common in polymyositis, but rare in muscular dystrophy.

(iv) In polymyositis weakness often exceeds the wasting of muscles, and in the early stages the reflexes are preserved; in dystrophy the wasting usually parallels the degree of weakness, pseudohypertrophy is common, and the reflexes disappear early in the disease (Walton, 1961).

Investigations are invaluable in establishing the diagnosis. The ESR is sometimes elevated, but this is inconstant; an elevated gamma globulin fraction of serum proteins associated with a decreased albumin fraction (Gavrilescu and Small, 1962) is found in over half the cases.

Electromyography (EMG) reveals lowamplitude polyphasic action potentials, on maximal voluntary contraction indicating a myopathy. Fibrillation and 'saw tooth' potentials, and a pseudomyotonic pattern are also frequently seen, and are thought to be associated with muscle fibre irritability. Electromyography is however a random sampling method, and normal patterns may be obtained for this reason, unless many different areas are sampled. With this reservation, EMG studies show evidence of myopathy in $80 \%$ of patients.

Serum aldolase and SGOT are elevated in about half the patients, especially those with the most active disease (Barwick and Walton, 1963). Serum creatine kinase is elevated in only one third of cases. (Pearce, Pennington and Walton, 1964). These changes are helpful in distinguishing polymyositis from endocrine and metabolic myopathies in which normal values are usual. Serum creatine kinase estimations are of limited value in separating polymyositis from dystrophy unless a small elevation or normal level are found, which would favour polymyositis.

Muscle biopsy should be performed on a proximal muscle - e.g., deltoid or quadriceps, in which EMG studies have not been performed. The typical changes consist of (1) Primary degeneration or necrosis of muscle fibres, (g) basophilic areas of regeneration with swollen vesicular nuclei, (3) infiltration of inflammato cells. Varying degrees of phagocytosis and interstitial fibrosis are also common.

Treatment consists of high doses of preef nisone, commencing with $20 \mathrm{mg}$. tds, which should be reduced very slowly only whe considerable clinical improvement has occurreo It may take up to 2-3 months before ameliora tion commences. Thereafter the drug shouidt be continued for 1-2 years before it is totally withdrawn. If a relapse should occur, the dose has to be increased until the symptoms ares again controlled. Occasionally ACTH 60-89 Units daily by intramuscular injection wi afford a remission unobtainable by prednisone. In a series of 52 patients treated by Barwick and Walton (1963), 34 recovered or improvedit and the remainder were unchanged, worse, of died. This included Group IV patients with malignancy, all of whom fared badly as antî cipated.

\section{Polymyalgia Rheumatica}

This is probably an inflammatory disorde $\frac{\check{c}}{5}$ of the intramuscular connective tissue, not in $=$ volving muscle fibres (Walton, 1964). B 8 \% patient is over the age of 60 , and has severes, unrelenting aching pains around the neôk. shoulders and upper arms, sometimes also $\overline{\mathrm{mn}}$ volving the buttocks and thighs. Movements of shoulder and hip joints are limited, bukू solely by pain, not by muscular weakness oB arthritis. Physical examination is otherwise negative, but the ESR is invariably oves $50 \mathrm{~mm} / \mathrm{hr}$, and a mild normochromic anæmią is a common accompaniment. (Gordon, 1960? Bagratuni, 1963). High levels of plasma fibrinogen $(>400 \mathrm{mg} . / 100 \mathrm{ml}$.) and of alpha -3 2 globulin values are frequently found. Alestig. and Barr (1963) found evidence of arteritis if the temporal arteries in seven out of ten cases? and 16 of 21 biopsies of the temporal arteries showed similar signs of arteritis in a series 9 reported by Hamrin, Jonsson, and Landberg (1964). These findings suggest that giant cello arteritis and polymyalgia rheumatica are twon. clinical variants of the same disease-an 9 arteritis of the elderly. The response to pred- $N$ nisone is dramatic in all patients with poly-岕 myalgia, and spontaneous remission occurso within approximately two years.

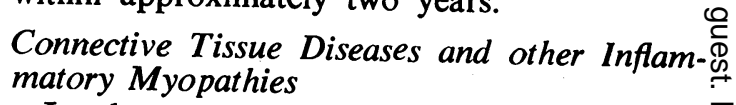
Involvement of muscle is a fairly common pathological finding in many of the so called $\underset{\nabla}{\circ}$ 
'auto-immune' collagen diseases (Sokoloff, Wilens, Bunim and McEwen, 1950). Signs of myopathy are however infrequent. A chronic progressive proximal amyotrophy is seen in Sjögren's syndrome, and a 'focal nodular myositis' is found in biopsies of muscle in patients with systemic lupus erythematosus, systemic sclerosis, rheumatoid disease, and polyarteritis nodosa, These pathological lesions are sometimes however, unaccompanied by clinical signs of myopathy, and the histological changes are not of value in differentiating the different primary collagen disease with which they are associated. A curious 'vacuolar myopathy' is occasionally seen in systemic lupus erythematosus (Pearson and Yamazaki, 1958) and appears to be specific. Ischæmic lesions of muscle due to an underlying arteritis may produce patchy and asymmetrical weakness, wasting and tenderness of muscles in any of these disorders. The typical non-caseating granulomata of sarcoidosis can occur in the muscles of patients with evidence of systemic sarcoidosis and a proximal myopathy, which clinically resembles chronic polymyositis has been described in such patients (Crompton and MacDermot, 1961). The pathological sarcoid granulomata, are however much more common in patients with no clinical signs of myopathy and indeed may afford a useful aid to diagnosis, in conjunction with the clinical signs, biopsy findings from glands and liver and the Kveim test.

Although it can be seen that focal pathological lesions in the muscles of patients with the diseases associated with disordered immunemechanisms are fairly frequent, it will be appreciated that such lesions are often nonspecific in type. The clinical picture is of a proximal myopathy, usually in an ill patient, which may be accompanied by a polyneuropathy, or mononeuritis multiplex, as well as by evidence of multiple system involvement, a high ESR abnormal electrophoresis, and sometimes by findings indicative of the underlying disease, e.g., LE cells, Waaler-Rose tests. The occurrence of such lesions, and the clinical overlap between the diseases which they complicate and polymyositis is striking, and is at least suggestive that polymyositis itself falls into this group of disorders.

\section{Metabolic Myopathies}

\section{Familial Periodic Paralysis}

Patients afflicted by this group of disonders occur sporadically, or may inherit the trait as an autosomal dominant with a predilection for males. The hypokalæmic variety is characterised by attacks of flaccid paralysis of the limbs and trunk but sparing the muscles of smiling, swallowing, talking and breathing. The attacks commence in the second decade, but decrease in frequency after the middle thirties. Paralysis may be partial or complete, and lasts from 6-24 hours, followed by total recovery. Attacks are precipitated by rest after exercise, a high carbohydrate meal, anxiety and cold. During the attack the serum potassium falls to below $3 \mathrm{mEq} / 1$, and it has been shown that potassium moves into the muscle cells and this is associated with dilatation of the endoplasmic reticulum (Shy, Wanko, Rowley and Engel, 1961). Attacks may be induced by administering insulin and glucose. The current view is that this disorder is due to an inherited enzyme defect resulting in an excessive accumulation of indiffusible products of carbohydrate metabolism with a consequent shift of potassium and water into the cells (McArdle, 1956, 1964). Treatment consists of intraveneous potassium chloride $(5-15 \mathrm{gm})$ for the acute attack, and oral potassium salts, or spironolactone as interval therapy.

Hyperkalæmic periodic paralysis (Gamstorp, 1956) usually begins in the first decade, and the attacks are shorter in duration, lasting for less than two hours. The pattern is similar, but attacks are precipitated immediately after exercise, or by ingestion of food with a high potassium content. Some patients show evidence of myotonia, which may be limited to the ocular muscles (Van't Hoff, 1962) relating this condition to paramyotonia. During an episode of paralysis, the serum potassium is elevated to more than $5 \mathrm{mEq} / 1$ but weakness may occur at lower levels. Evidence exists (MoArdle, 1962; Gamstorp, 1962) that there is a leak of potassium from muscle cells, and myopathic changes are seen electromyographically during an episode of weakness; furthermore it has been suggested that an abnormally low resting membrane potential is associated with, though not necessarily caused by the leakage of intracellular potassium.

Treatment consists of chlorothiazide $0.5 \mathrm{~g}$. daily to produce a deficit of body potassium; dichlorphenamide $50 \mathrm{mg}$ daily is also most effective in reducing the frequency and severity of attacks.

Poskanzer and Kerr (1961) have described a normokalæmic variety, with attacks lasting for several days. The serum potassium is normal, attacks were precipitated by oral potassium, 
but improvement occurred following large doses of salt, or of salt retaining steroids e.g., fludrocortisone, $0.1 \mathrm{mg}$. daily. The diagnosis of periodic paralysis can usually be made on the history if other members of the family are involved. The precipitating factors described give a clue to the variety of potassium imbalance, and confirmation is obtained by inducing an attack and by assessment of the electrolytes and the electrophysiological state during such an attack. The response to therapy is a further useful point. It should be remembered that occasionally periodic paralysis may be symptomatic of thyrotoxicosis, and in such circumstances the attacks respond to the specific treatment of the underlying condition.

\section{Glycogen Storage Diseases of Muscle}

This group of conditions is characterised by a defect of one of the enzymes essential to normal glycogen metabolism. At least six varieties are known (Thomson, MacLaurin and Prineas, 1963), and they may involve the liver, kidney, myocardium or skeletal muscle. In children with muscular involvement, profound weakness and hypotonia are apparent usually from infancy. Adult patients have more recently been described who present with weakness of the limbs and muscle pains after exercise, or with extremes of temperature (Holmes, Houghton and Woolf, 1960). Investigations show a failure of elevation of blood lactic acid after ischæmic exercise of the limb, and abnormalities of anærobic glycolysis of muscle homogenates can be demonstrated in vitro on biopsy material. Abnormal collections of PAS positive glycogen can be seen in subsarcolemmal aggregations in muscle biopsies.

The best known of this group is McArdle's Syndrome (McArdle, 1951). He reported a 30year-old man who suffered from muscular pain, weakness and stiffness after exercise which was relieved by rest. After repeated gripping with his hands he was unable to extend the fingers. These symptoms dated from childhood. Subsequent cases have been reported, some with occasional myoglobinuria. In these patients there exists a failure of the normal three to fourfold increase of blood pyruvate and lactate, following ischæmic exercise, which together with the subsarcolemmal aggregations of glycogen help to confirm the diagnosis. Such patients may be misdiagnosed as having peripheral vascular disease, sciatica, or psychoneurosis. Schmid and Hammaker (1961) have shown that McArdle's syndrome is due to a single completely recessive autosomal gene, and a specific deficiency of myophosphorylase has been co clusively demonstrated in these patients (Peaz son, Rimer and Mommaerts, 1961). Or\$1 fructose, $50-100 \mathrm{~g}$. prior to exercise diminishes the symptoms. The condition is probably nof progressive in the absence of permanent muscular weakness and wasting, and in patiengs without myoglobinuria.

\section{Myoglobinuric Myopathies}

Myoglobin in the urine renders the colour red to chocolate brown; the urine gives a posi. tive guaiac test, but is not fluorescent, and contains no red cells. These features distinguispos myoglobinuria from hæmoglobinuria, porphyo ria, and hæmaturia. Myoglobinuria may occlo transiently in any acutely destructive lesion of muscle and is most frequently seen in crush injuries, after extreme exertion, and in HaAf disease-a toxic myolysis following ingestion of fish contaminated by an unidentified toxint in Germany.

Idiopathic Paroxysmal Myoglobinuria pro duces acute attacks of cramp, pain, weaknessand tenderness of skeletal muscles, which whe severe can produce prostration and respiratory paralysis (Borman, Davidson and BlondheBin 1963). Attacks last 2-3 days and are precipitated? by exercise, or by infection. Rest and alkaliāio sation of the urine are the only important point in therapy, unless acute renal failure occurs which merits therapy in its own right.

\section{Myopathies Associated with Electrolyte Imbalance}

Hypokalæmia may be secondary to diarrhoea thiazide diuretics, steroids, diabetic coma? aldosteronism, and to a potassium-losings nephritis. The usual clinical features are apathy? delirium, or stupor, muscle twitching, and car diac arrhythmias. In severe hypokalæmia a widespread flaccid paralysis of all muscles may occur; in the early stages this may be curiously? selective in distribution, and the reflexes ares, preserved; in a short space of time a tota areflexic flaccid tetraparesis results. A low: serum potassium, and the typical ECG changeso confirm the diagnosis.

Hyperkalæmic paralysis may occur in acute oliguric renal failure, or following massive in $-\omega$ travascular hæmolysis. A flaccid paralysis with? loss of reflexes is usually of fairly sudden on $\frac{0}{6}$ set: speech, swallowing, and respiration may be involved, and in the early stages a curious ${ }^{+}$ mental attitude of hostility and resentment is $\frac{0}{0}$ observed. The cardiac muscle is invariably affected and cardiac arrhythmias and arrest are ${ }_{\mathbb{\Phi}}$ 
the usual cause of death. Intravenous calcium gluconate or insulin and glucose should be given urgently when the diagnosis has been confirmed by the estimation of serum potassium, and by the ECG.

A proximal myopathy with preservation of reflexes has been observed in hyperparathyroidism, and in renal tubular acidosis (Henson, 1965). A similar clinical picture has been recorded in hypocalcæmic states of varied ætiology, and these conditions can closely resemble chronic polymyositis. (Richet, Sourdel and Percola, 1937; Pearce, 1965).

\section{Unclassified Myopathies}

Central Core Disease (Shy and Magee, 1956; Engel, Foster, Hughes, Huxley and Mahler, 1961) is a curious non-progressive myopathy producing hypotonia, weakness and impaired motor skills in infancy. The centre of the muscle fibres show a dense amorphous cylinder of PAS positive material, which has been shown to be lacking in oxidative enzymes and phosphorylase. There is little doubt this is a real entity, but its place in the classification of congenital myopathies is uncertain.

Nemaline myopathy has been recorded on two occasions. The clinical picture is of a proximal myopathy with involvement of spinal and abdominal muscles, but a less rapid progression than seen in muscular dystrophy. Both cases ((Engel, Wanko, and Fenichel, 1964) have shown in the smaller muscle fibres, collections of rod-shaped fibrils of a protein material, related to myosin. The natural history and nosological status of nemaline myopathy are unknown.

\section{Endocrine Myopathies}

\section{Thyroid Myopathies}

Thyrotoxicosis in the untreated state is frequently accompanied by weakness and sometimes wasting of the muscles of the shoulder and pelvic girdles. Difficulty in lifting objects to high shelves, trouble in climbing stairs, and rising from low chairs are common complaints. These focal muscular symptoms are easily masked by the general fatigue of the patient, but clinical signs and a myopathic pattern on electromyography are found in $80 \%$ of patients (Havard, Campbell, Ross and Spence, 1963). In a series of 240 thyrotoxic patients, Satoyoshi and his colleagues (1963) found moderate or severe weakness in $60 \%$, and gross focal wasting in $7 \%$. Clinical signs were most frequent in patients over the age of 40 , and in those with hyperthyroidism of longest duration.
It is clear that the electromyographic and pathological abnormalities are not constantly related to the severity of the myopathy. This is probably due to the patchy distribution of the myopathic process, and the random techniques of sampling which are utilised. Granular degeneration, œdema and cellular infiltration of connective tissue, have been reported (Satoyoshi and others, 1963) but frequently only non-specific changes are seen (Adams, DennyBrown, and Pearson, 1963). A complex metabolic defect, involving catabolism of all body protein reserves, and a separate specific inhibitory action of thyroxine upon muscle creatine kinase, are probably responsible for chronic thyrotoxic myopathy. Treatment of the thyrotoxicosis totally reverses the myopathy, a fact in contrast to the effect of treatment upon endocrine exophthalmos. The cause of this latter condition is obscure but is probably related to a specific 'exophthalmos producing substance' secreted by the anterior pituitary. Seen in association with pretibial myxœdema and thyroid acropachy, it tends to progress most rapidly after thyroidectomy or antithyroid drug therapy, especially in male patients over the age of 40 . Some response to large doses of thyroxine and prednisone is usual, but irradiation of the pituitary, orbital decompression, or hypophysectomy have to be considered, in conjunction with tarsorrhaphy in the refractory case, to avoid corneal ulceration.

Thyrotoxicosis is a rare cause of myasthenia in young people (Drachman, 1962). The thyrotoxicosis develops before, or at the same time as the myasthenia, and one remarkable instance of the two diseases occuring in sisters has been recorded (Greenberg, 1964). It seems likely that the disorder glorified by the name of 'acute thyrotoxic myopathy' is in fact thyrotoxicosis and the bulbar syndrome of thyrotoxic myasthenia. Both conditions require specific therapy, but surgery in the myasthenic patient is a calculated hazard. Pericdic paralysis has been mentioned as an occasional complication of hyperthyroidism which responds to treatment of the primary disease.

A myopathy is an occasional occurrence in hypothyroidism, in which however pseudomyotonic tendon jerks are very common. The myopathy is characterised by diffuse weakness, slowness of movement and enlargement of affected muscle groups. In the adult this is known as Hoffman's syndrome, and despite the pseudomyotonic reflexes, there is no electrical evidence of myotonia, and there are no constant pathological changes in muscle biop- 
sies, (Adams, Denny-Brown and Pearson, 1963). Thyroxine in adequate doses reverses this myopathy.

\section{Pituitary and Adrenal Myopathies}

In the early stages of acromegaly, there occurs a hypertrophy and increase in power of skeletal muscles. In the later stages, when hypopituitarism supervenes, generalised muscular weakness is common. It has been claimed that characteristic crescents, or 'half-moons' are seen in specially stained sections from muscle biopsies in acromegalics, and in patients with thyroid disorders (Kirchheiner, 1962). These half moons are thought to be collections of muco-polysaccharides, and have been related to the increased thyrotropin stimulation in these diseases. These findings have yet to find general acceptance.

A proximal myopathy is common in Cushing's Syndrome. Weakness and wasting of proximal muscles and diminished reflexes are seen, and are accompanied by the well known cutaneous atrophy, bruising, striæ, and truncal obesity. Since Muller and Kugelberg (1959) described this syndrome, many patients treated with corticosteroids have exhibited identical features. Steroid myopathy (reviewed by Golding, Murray, Pearce and Thompson, 1961) is related largely to the dose of steroid given and the duration of the therapy. However it is particularly common following the administration of the 9-alpha-fluorinated steroids triamcinolone, dexamethasone, and fludrocortisone; electromyography shows a slight reduction of the interference pattern, increased numbers of small potentials with reduced amplitude and duration, and increased numbers of short duration polyphasic potentials. Histology shows remarkably little abnormality. Cessation of steroid therapy has in the writer's experience been invariably accompanied by total recovery.

It has been alleged that there exists a myopathy in rare examples of Addison's disease with a specific tendency to develop flexion contractures. It seems probable that these signs are due to a biochemical disorder of tendons and fascia, and not to any specific myopathy.

In all the endocrine myopathies, serum creatine kinase levels are normal, and the diagnosis is not difficult if the underlying disease is detected. (Pearce and others, 1964).

\section{Neoplastic Myopathy}

Since the pathological finding of a myopathy in two patients with carcinoma of the lung, reported by Denny Brown in 1948, a group of non-metastatic neurological syndromes associo ated with neoplasm have been reported. These include (Brain, 1963; Croft and Wilkinson? 1963; Mancall and Rosales, 1965), the follow:ing syndromes: -

1. A subacute cerebellar syndrome

2. A pure sensory, pure motor, or mixees polyneuropathy

3. Progressive dementia or depression

4. Progressive multifocal leucoencephalo pathy-usually associated with a malige nant lymphoma

5. Acute necrotising myelopathy

6. The myopathic - myasthenic syndrome

The myopathy is characterised by an atros phic paresis, most marked in the proximas muscles of the limbs, and trunk. Pelvic or shoulder girdle muscles may be affected, and often both. A lordotic waddling gait has been recorded, and occasionally weakness of the neck muscles is present. (Henson, Russelle and Wilkinson, 1954). The tendon reflexeg are depressed, and fasciculation is not seen. The condition is insidious in evolution, and downhill progression may on occasion be interrupted by spontaneous but fleeting $\mathrm{im}_{-}$ provement. The myopathy commonly aniees dates the presentation of the neoplasm, or nas follow it months or years later.

A proportion of these patients exhibit myäss thenic symptoms of muscle fatiguability. AD partial response to neostigmine is seen, bu* this is far less impressive than the dramati\& response in myasthenia gravis. Such patients are abnormally sensitive to decamethonium t $\Theta$ which cases of myasthenia gravis are resistante Potentiation of muscle power, following exer? cising through a period of fatigue ("seconde wind phenomenon") is a curious finding. It iṣ. accompanied electrically, by a post-tetanic in crement in the amplitude of evoked action potentials produced by stimulating moto? nerves at rates of more than 50 per second.

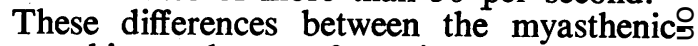
myopathic syndrome of carcinoma and myas? thenia gravis should be sought in patients. presenting with myasthenia-particularly ing later life. Similarly, a carcinomatous myopath $\Varangle$ without myasthenia may closely mimic poly: myositis. The current view is that this syncw drome is a reaction of muscle tissue to are auto-immune mechanism directed against at antigen in the muscle or in tumour tissue definitive proof of this aetiological hypothesis is however still lacking. Of the contributor factors, the age of the patient, the duration of symptoms of carcinoma, and the bloo of 
group have been investigated, and found not to be related to the incidence of neuromyopathy in patients known to be suffering from breast or lung cancer (Croft and Wilkinson, 1963).

The pathological changes are disproportionately slight compared to the extent of the clinical signs. Minimal fibre atrophy with phagocytosis and nuclear chains, is all that is usually seen. The nerve endings are quite different from those seen in myasthenia gravis. The commonest tumour implicated in the myopathic-myasthenic syndrome is a bronchogenic carcinoma. Carcinoma of breast, ovary, gastrointestinal tract, kidney, and occasionally lymphomas, may produce an identical picture. The prognosis is from a few months to $2-3$ years, and treatment is directed at the primary growth.

\section{The Myasthenic Syndrome}

Myasthenia is an abnormal fatiguability of muscle. The commonest cause is myasthenia gravis, but as mentioned above, this symptom may be secondary to thyrotoxicosis, and also occurs in the myopathic-myasthenic syndrome associated with carcinoma. Myasthenia gravis is dealt with elsewhere in this symposium, but some points of diagnostic importance are germane to this discussion.

The pathological changes in myasthenia gravis are relatively sparse. They consist of focal coagulative necrosis of one or many fibres, collections of lymphorrhages and simple fibre degeneration. These changes are not specific to myasthenia (Russell, 1953). Proliferation of minute nerve fibres from the knobs of the terminal nerve fibres at the motor point, has been demonstrated in myasthenics, but the full significance of this finding is uncertain (Woolf, 1963). These features are usually easy to differentiate from the pathological picture seen in other myopathies.

Myasthenia gravis may present at any age from the neonatal period to late middle-life. It most frequently starts in the ocular muscles producing ptosis and diplopia with normal pupillary size, The ocular muscles are involved in $90 \%$ of patients, next in frequency are the flexors and extensors of the neck, proximal muscles of the shoulder and pelvic girdles, and muscles of facial expression, chewing and swallowing, and phonation. Myasthenia may remain localised to any one of the above groups throughout the patient's life, or may spread from one site to involve another after many years.

The cardinal feature is the fatiguability, producing weakness after repeated muscle contraction, commonly worse at the end of the day. Ap effort should be made to induce such weakness by repeatedly exercising the appropriate muscles.

The observed weakness is reversed within 2 minutes by intravenous edrophonium (10 mg. diluted in $5 \mathrm{ml}$. saline), or within 30 minutes by neostigmine $1.5 \mathrm{mg}$., i.m. Caution is needed when using these tests in a treated but relapsed patient, as a cholinergic crisis (neostigmineresistant) may be imminent. A curious differential effect of neostigmine is not uncommon, in which ocular signs are reversed, but bulbar muscles or limb muscles become weaker after the injection, or after oral medication. A late sequel to myasthenia is a true myopathic wasting and weakness which is not totally reversed by drugs.

In an untreated patient, electromyography shows a characteristic decrement of evoked action potentials on repetitive supramaximal stimulation of the motor nerve (Simpson, 1960).

The ætiology of this syndrome is still unknown. There is a significant association with connective tissue disorders-especially rheumatoid arthritis, thyroid diseases, disseminated lupus erythematosus, and pernicious anæmia (Simpson, 1965). The response to thymectomy (Simpson, 1958) in some patients is also suggestive of an auto-immune mechanism, since the role of the thymus in this group of diseases is certainly an important one, even though many details of the mechanisms involved are still obscure. A thymoma is present in about $10 \%$ of patients who are usually difficult to control.

\section{Conclusions}

In this account I have endeavoured to mention the salient features of the more common acquired myopathies, and the technical details of research procedures have been deliberately omitted. The muscle appears to be a target for many acquired systemic diseases, and it is only capable of reacting in certain ways governed by its intrinsic morphology and function. Thus, both clinical signs and histological, biochemical and electrophysiological studies tend not to be specific for any one disease. However a combination of all these methods, and particularly the clinical background, and the clinical evidence of other systemic manifestations of the primary disease will almost always allow the physician to make the correct diagnosis. This is particularly rewarding in acquired myopathies, because 
specific therapy frequently produces amelioration of the patient's symptoms.

I am deeply indebted to Dr. John Walton, who stimulated my interest in muscle diseases, and has so freely given of his expert knowledge in this field.

\section{REFERENCES}

Adams, R. D., Denny-Brown, D., and Pearson, C. M. (1963): Diseases of Muscle. 2nd Ed. London: Henry Kimpton.

Alestig, K., and BARR, J. (1963): Giant Cell Arteritis, Lancet, i, 1228.

Bagratuni, L. (1963): Prognosis in the Anarthritic Rheumatoid Syndrome, Brit. med. J., i, 513.

Barwick, D. D., and Walton, J. N. (1963): Polymyositis, Amer. J. Med., 35, 646.

BormaN, J. B., DAvidson, J. T., and Blondheim, S. H. (1963): Idiopathic Rhabdomyolysis, as an Acute Respiratory Problem, Brit. med. J., ii, 726.

BraIN, W. R. (1963): The Neurological Complications of Neoplasms, Lancet, i, 179.

Croft, P. B., and Wilkinson, M. (1963): Carcinomatous Neuromyopathy, Lancet, i, 184.

CROMPton, M. R., and MACDERMOT, V. (1961): Sarcoidosis Associated with Progressive Muscular Wasting and Weakness, Brain, 84, 62.

DENNY-Brown, D. (1948): Primary Sensory Neuropathy with Muscular Changes Associated with Carcinoma, J. Neurol. Neurosurg. Psychiat., 11, 73.

Drachman, D. B. (1962): Myasthenia Gravis and the Thyroid Gland, New Engl. J. Med., 266, 330.

ENGel, W. K., Foster, J. B., Hughes, B. P., Huxley, H. E., and MAHLER, R. (1961): Central Core Disease, Brain, 84, 167.

ENGEL, W. K., WANKo, T., and Fenichel, G. M. (1964): Nemaline Myopathy, Arch.Neurol. (Chic.), 11, 22.

Gamstorp, I. (1956): Adynamia Episodica Hereditaria, Acta. paediat. (Uppsala) Suppl., 108, 1.

GAMSTORP, I. (1962): A Study of Transient Muscular Weakness, Acta. psychiat. (Kbh), 38, 3.

Gavrilescu, K., and Small, J. M. (1962): Serum Electrophoretic Change in Polymyositis, Brit. med. J., 2, 1720.

Golding, D. N., Murray, S. M., Pearce, G. W., and ThOMPSON, M. (1961): Corticosteroid Myopathy, Ann. phys. Med., 6, 171.

GoRDON, I. (1960): Polymyalgia Rheumatica, Quart. J. Med., 116, 473.

GrEeNBER̈G, J. (1964): Myasthenia Gravis and Hyperthyroidism in Two Sisters, Arch. Neurol. (Chic.), 11, 219.

HAMrin, B., JonsSON, N., and LANDBERG, T. (1964): Arteritis in Polymyalgia Rheumatica, Lancet, $\mathbf{i}$, 397.

Havard, C. W. H., Campbell, E. D. R., Ross, H. B., and SPENCE, A. W. (1963): Electromyographic and Histological Findings in the Muscles of Patients with Thyrotoxicosis, Quart. J. Med., 32, 145.

Henson, R. A., Russell, D. S., and Wilkinson, $M$. (1954): Carcinomatous Neuromyopathy. A Clinical and Pathological Study, Brain, 77, 82.

HENSON, R. A. (1965): Research in Muscular Dystrophy. 3rd Symposium. London: Pitman.

Holmes, J. M., Houghton, C. R., and Woolf, A. L. (1960): A Myopathy Presenting in Adult Life with Features Suggestive of Glycogen Storage Disease, J. Neurol. Neurosurg. Psychiat., 23, 302.
KIRCHHEINER, B. (1962): Specific Muscle Lesions Pituitary-thyroid Disorders, Acta med. scand., 172, 539.

Mancall, E. L., and Rosales, R. K. (1965): Necr@ tizing Myelopathy associated with Visceral Carcinoma, Brain, 87, 639.

MCARDLE, B. (1951): Myopathy due to a Defect i Muscle Glycogen Breakdown, Clin. Sci., 10, 13.

McArdle, B. (1956): Familial Periodic Paralysiç Brit. med. Bull., 12, 226.

MCARDLE, B. (1962): Adynamia Episodica Hered taria, Brain, 85, 121.

MCARDLE, B. (1964): In Disorders of Voluntarys Muscle, ed. J. N. Walton, Chap. 15. London? J. \& A. Churchill.

Muller, R., and Kugelberg, E. (1959): Myopathy in Cushing's Syndrome, J. Neurol. Neurosur\& Psychiat., 22, 314.

Pearce, J. M. S., Pennington, R. J., and Walto J. N. (1964): Serum Enzyme Studies in Musctg Disease. Part 2, ibid, 27, 96.

PeARCE, J. M. S. (1965): Research in Musculaf Dystrophy. 3rd Symposium. London: Pitman. is

Pearson, C. M., and Yamazaki, J. N. (1958) Vacuolar Myopathy in Systemic Lupus Erytheso matosus, Amer. J. clin. Path., 29, 455.

PeArson, C. M., Rimer, D. G., and Mommaerts W. F. H. M. (1961): A Metabolic Myopathy due to Absence of Muscle Phosphorylase, Amer. Med., 30, 502.

PEARSON, C. M. (1964): In "Disorders of Voluntar Muscle," ed. Walton, J. N., Chapter 12. London? J. \& A. Churchill.

Poskanzer, D. C., and KerR, D. N. S. (1961). Third Type of Periodic Paralysis with Normekalaemia and Favourable Response to Sodining Chloride, Amer. J. Med., 31, 328.

Richet, C., Sourdel, M., and Percola, A. (1937)공 Syndromes Parathyroidmusculaires, J. méd. françn్ 26, 377.

Russell, D. S. (1953): Histological Changes in the Striped Muscles in Myasthenia Gravis, J. Pat $\vec{F}$ Bact., 65, 279.

Satoyoshi, E., Murakami, K., Kowa, H., Kinoshita M., Noguchi, K., Hoshina, S., NishiYama, Y., ang ITO, K. (1963): Myopathy in Thyrotoxicosis; Neurology (Minneap.), 13, 645.

SCHMID, R., and HAMMAKER, L. (1961): Hereditar Absence of Muscle Phosphorylase, New Engl. Med., 264, 223.

Shy, G. M., and Magee, K. R. (1956): A New Non-progressive Myopathy, Brain, 79, 610.

Shy, G. M., Wanko, T., Rowley, P. T., and EngeL, A. G. (1961): Studies in Familial Periodic Paralysip̧ Exp. Neurol., 3, 53.

SimpSON, J. A. (1958): An Evaluation of Thymectomw in Myasthenia Gravis, Quart. J. Med., 81, 112.

Simpson, J. A. (1960): Myasthenia Gravis: a Nege Hypothesis, Scot. med. J., 5, 419.

SIMPSON, J. A. (1965): Immunological Disturbances in Myasthenia Gravis with a Report of Hashimotols Disease Developing after Thymectomy, J. Neurof Neurosurg. Psychiat., 27, 485.

SokolofF, L., Wilens, S. L., Bunim, J. J., McEweP C. (1950): Diagnostic Value of Histologic Lesions of Striated Muscle in Rheumatoid Arthritis, Ame J. med. Sci., 219, 174.

Thomson, W. H. S., Maclaurin, J. C., and Prineaș J. W. (1963): 'Skeletal Muscle Glycogenosis J. Neurol. Neurosurg. Psychiat., 26, 60. 
Van't Hoff, W. (1962): Familial Myotonic Periodic Paralysis, Quart. J. med., 31, 385.

Walton, J. N., and ADams, R. D. (1958): Polymyositis. Edinburgh and London: E. \& S. Livingstone.

Walton, J. N. (1961): Muscular Dystrophy and its Relation to the Other Myopathies, Res. Publ. Ass. nerv. Ment. Dis., 38, 378.
Walton, J. N. (1964): Some Diseases of Muscle, Lancet, i, 447.

Woolf, A. L. (1963): Changes in the Terminal Motor Innervation in Cases of Neuromuscular Disease in "Research in Muscular Dystrophy". 2nd Symposium, p. 99. London: Pitman. 